\title{
Inversion of Sequence of Diffusion and Density Anomalies in Core-Softened Systems
}

\author{
Yu. D. Fomin, E. N. Tsiok, and V. N. Ryzhov \\ Institute for High Pressure Physics, Russian Academy of Sciences, Troitsk 142190, Moscow Region, Russia
}

(Dated: March 2, 2022)

\begin{abstract}
In this paper we present a simulation study of water-like anomalies in core-softened system introduced in our previous publications. We investigate the anomalous regions for a system with the same functional form of the potential but with different parameters and show that the order of the region of anomalous diffusion and the region of density anomaly is inverted with increasing the width of the repulsive shoulder.
\end{abstract}

PACS numbers: 61.20.Gy, 61.20.Ne, 64.60.Kw

\section{INTRODUCTION}

It is well known that some liquids demonstrate anomalous behavior in some regions of thermodynamic parameters. The most common and well known example is water. The water phase diagrams have regions where a thermal expansion coefficient is negative (density anomaly), self-diffusivity increases upon compression (diffusion anomaly), and the structural order of the system decreases with increasing pressure (structural anomaly) [1-6]. Later on it was discovered that many other substances also demonstrate similar behavior. Some typical examples are silica, silicon, phosphorus and many others [5-20].

It is reasonable to relate this kind of behavior to the orientational anisotropy of the potentials, however, a number of studies demonstrate water-like anomalies in fluids that interact through spherically symmetric coresoftening potentials with two length scales (see, for example, the reviews [21] and 22]). A lot of different coresoftened potentials were introduced. All such systems can be approximately divided into two classes: shoulderlike, composed of a hard-core and a repulsive shoulder, softening the core, and ramp-like systems, composed of a hard-core and a repulsive ramp, establishing two competing equilibrium distances [21, 22]. Anomalous behavior usually takes place in ramp-like systems, while it is not observed in purely shoulder-like ones. However, if instead of "hard" step one considers a smoothed one the anomalies appear (see, for example, 21 24, 57 61].

As it was found in experiments 1] and simulations [3, 4], the water anomalies have a well-definite sequence: the regions where these anomalies take place form nested domains in the density-temperature [3] (or pressuretemperature [4]) planes: the density anomaly region is inside the diffusion anomaly domain, and both of these anomalous regions are inside a broader structurally anomalous region. This water-like behavior was found in systems with core-softening potentials $21,23,2561$. However, in other anomalous systems the sequence of anomalies may be different. For example, the hierarchy of anomalies for silica is different compared to water. In this case the diffusion anomaly region contains the struc- tural anomalous region which, in turn, has the density anomaly region inside [63]. To our knowledge this is the only example of such inversion of the order of the anomalies.

This paper presents a simulation study of anomalies in core-softened system introduced in our previous publications [57 61]. We investigate the anomalous regions for a system with the same functional form of the potential but with different parameters and show that the order of the region of anomalous diffusion and the region of density anomaly is inverted.

The article is organized as following: Section II presents the system and methods, Section III describes the results and gives theirs discussion and Section IV contains conclusions.

\section{SYSTEMS AND METHODS}

The system we study in the present simulations is Smooth Repulsive Shoulder System (SRSS) introduced in our previous publications [57 59]:

$$
U(r)=\varepsilon\left(\frac{\sigma}{r}\right)^{n}+\frac{1}{2} \varepsilon\left(1-\tanh \left(k_{0}\left(r-\sigma_{1}\right)\right)\right),
$$

where $n=14, k_{0}=10 . \sigma$ is "hard"-core diameter and $\sigma_{1}=1.35 ; 1.45 ; 1.55 ; 1.8$ is soft-core diameter.

In the remainder of this paper we use the dimensionless quantities: $\tilde{\mathbf{r}} \equiv \mathbf{r} / d, \tilde{P} \equiv P d^{3} / \varepsilon, \tilde{V} \equiv V / N d^{3} \equiv 1 / \tilde{\rho}$. As we will only use these reduced variables, we omit the tildes.

In Refs. [57, 59] it was shown that this system demonstrates anomalous behavior. A relation between phase diagram and anomalous regions was also discussed in these articles. Our later publications gave detailed study of diffusion, density and structural anomalies in this system 60 62.

We simulated the systems with four different step sizes $\sigma_{1}$ and monitored the change in phase diagram and anomalous regions with increasing the step width.

Importantly, in low temperature region the systems can demonstrate slow dynamics. In order to get reliable 
results in the whole temperature range we used parallel tempering technic 64]. The system with $\sigma_{1}=1.35$ and $\sigma_{1}=1.45$ were simulated at densities from $\rho=0.3$ till $\rho=0.8$ with step $\delta \rho=0.05$ ( $\delta \rho=0.025$ in the vicinity of anomalous regions) and at temperatures from $T=0.12$ till $T=0.8$. In the case of $\sigma_{1}=1.55$ the range of densities was $\rho=0.2-0.75$. Finally, the density range of $\rho=0.1-0.8$ was simulated for $\sigma_{1}=1.8$. Along every isochor we ran several parallel tempering runs with different temperatures. Lowe-Andersen thermostat was used during the equilibration time [65]. After equilibration the thermostat was switched off and the system evolved in $N V E$ ensemble for production. After the production a trial change of temperature was made. Many data points were collected along every isochor (more then 100). Using these data points we constructed 9 -th order polynomial approximation of internal energy, pressure and diffusion coefficient along isochors. These approximations were used in the following analysis.

In order to compute the excess entropy of the liquids we used thermodynamic integration method [64]. We computed free energy along a high temperature isotherm by integrating equation of state of the system. After that we computed the free energies along isochors by integrating the $U / T^{2}$ function, where $U$ is the internal energy 64]. Excess entropy was obtained as $S_{e x}=\frac{U-F_{e x}}{N k_{B} T}$.

\section{RESULTS}

Here we present the anomalous regions for the four systems studied.

$$
\sigma_{1}=1.35
$$

The phase diagram and anomalies in this system were already reported in our previous publications [57 60]. Here we summarize the previous results and place the anomalous regions on the phase diagram. We also think that it is necessary to repeat the results for this particular system for the sake of completeness.

Figs. 1 (a)-(c) show the diffusion coefficient, pressure and excess entropy for the system with $\sigma_{1}=1.35$. One can see that all three anomalies take place in the system. It is also evident that structural anomaly is more stable than the diffusion one since it disappears at higher temperatures.

Fig. 2 places the anomalies regions on the phase diagram of the system reported in Refs. [57, 58]. One can see that all three anomalous regions appear exactly after the low-density crystal bump. This allows to relate the anomalies to the phase diagram, i.e. the reason for the anomalies to appear can be the presence of the crystal bump inside the liquid phase. The principle question here is that liquids to the left from the bump and to the
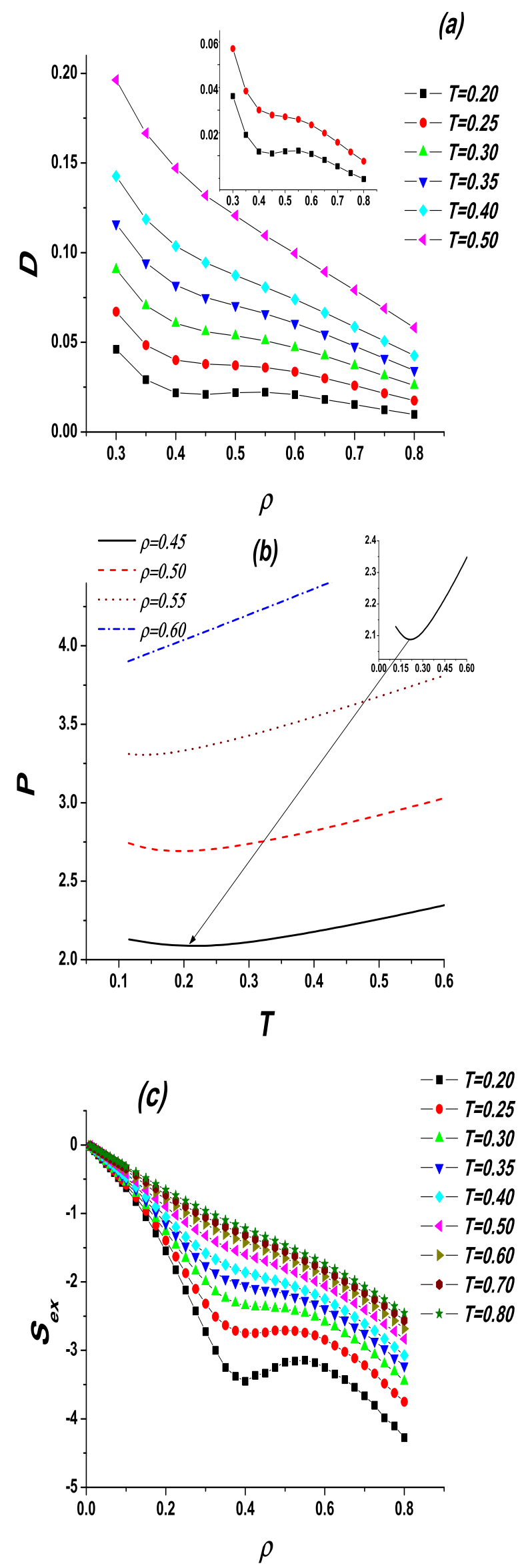

FIG. 1: (Color online). (a) Diffusion coefficient along a set of isotherms; (b) pressure along a set of isochors; (c) excess entropy along a set of isotherms for the system with $\sigma_{1}=1.35$. 


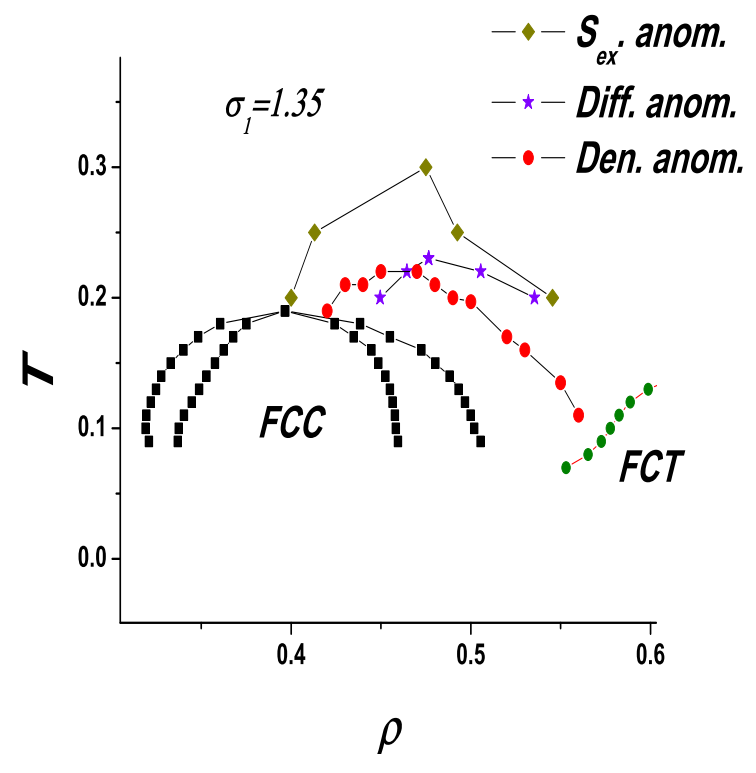

FIG. 2: (Color online). Location of anomalous regions at the phase diagram of the system with $\sigma_{1}=1.35$.

right have different local structures: while the highest radial distribution function peak at the "left" liquid is the second one, corresponding to the soft core distance, at the "right" liquid it is the first peak located on the hard core distance. In between of these two liquids a region appears where strong competition of two distances appears. It is this competition which brings to the appearance of anomalous behavior.

The second point to notice is that the anomalous regions correspond to the picture proposed for water [3], i.e. the diffusion anomaly region is inside the structural anomaly and the density anomaly is mainly inside the diffusion anomaly.

$$
\sigma_{1}=1.45
$$

Figs. 3 (a)-(c) show the anomalies for the system with $\sigma_{1}=1.45$. One can see from these pictures that the system behaves qualitatively similar to the previous case. However, the diffusion anomaly looks suppressed. At the temperatures as low as 0.15 the diffusion is close to a bend, but it is still monotonous. At the same time the density anomaly is rather pronounced in the system.

The location of anomalous regions and the low density Face Centered Cubic (FCC) phase for $\sigma_{1}=1.45$ system is shown in Fig. (4) One can see that the diffusion anomaly has almost gone under the melting line. Only a small part of diffusion anomaly region is located in stable liquid phase. At the same time density anomaly is still very pronounced. It occupies a large region in the liquid part of the phase diagram.
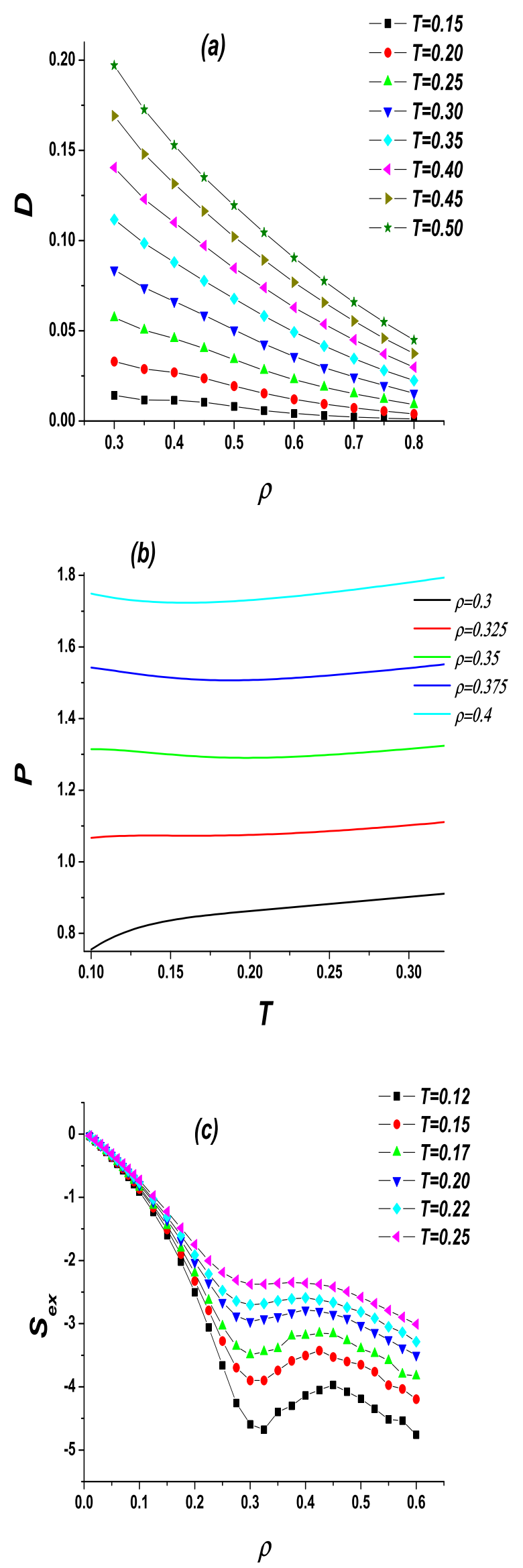

FIG. 3: (Color online). (a) Diffusion coefficient along a set of isotherms; (b) pressure along a set of isochors; (c) excess entropy along a set of isotherms for the system with $\sigma_{1}=1.45$. 


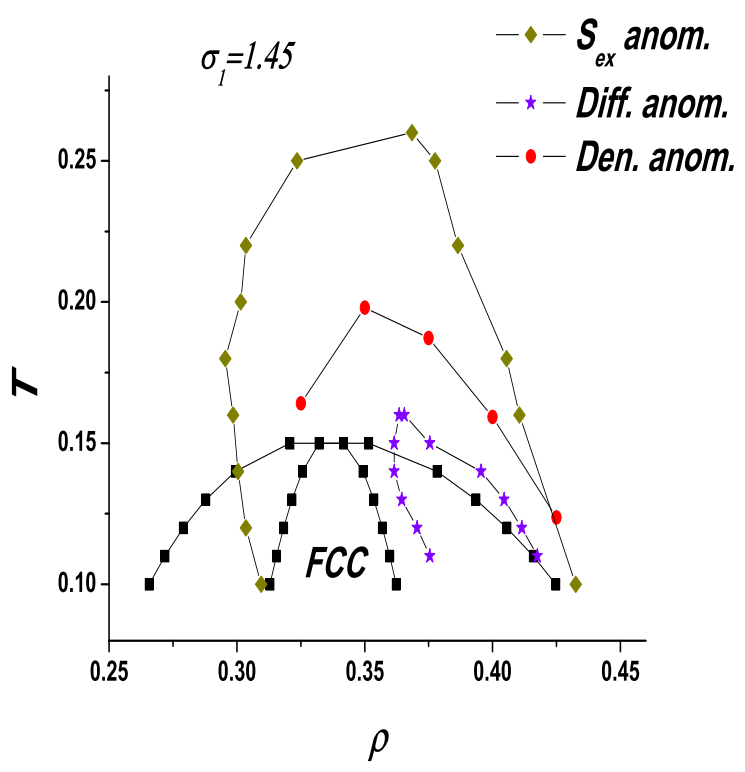

FIG. 4: (Color online). Location of anomalous regions at the phase diagram of the system with $\sigma_{1}=1.45$.

The most important fact extracted from Fig. 4 is that the diffusion and density anomalies inverted with respect to each other, i.e. now diffusion anomaly region is inside the density anomaly one. The similar inversion of the order of anomalies is found in silica, however, in silica one can see the inversion of the order of structural and diffusion anomaly [15]. We can conclude that the case of unusual sequence of anomalies in silica is not unique, and the SRSS system is one more example of such unusual behavior.

$$
\sigma_{1}=1.55
$$

In the case of $\sigma_{1}=1.55$ we do not find diffusion anomaly (Fig. 5(a)). At low temperatures the diffusion along an isotherm develops a bend, but not a loop. At the same time both density and structural anomalies are present in the system (Figs. 5. (b)-(c)). Comparing it with the results for the previous two cases we conclude that the diffusion anomaly is completely under the melting line for the present case which makes it unobservable.

From Fig. 6] we see that the density anomaly also approaches the melting line. This allows to suggest that density anomaly also shrinks with increasing the step size.

$$
\sigma_{1}=1.8
$$

In the case of $\sigma_{1}=1.8$ both diffusion and density anomalies are absent. Only excess entropy demonstrates

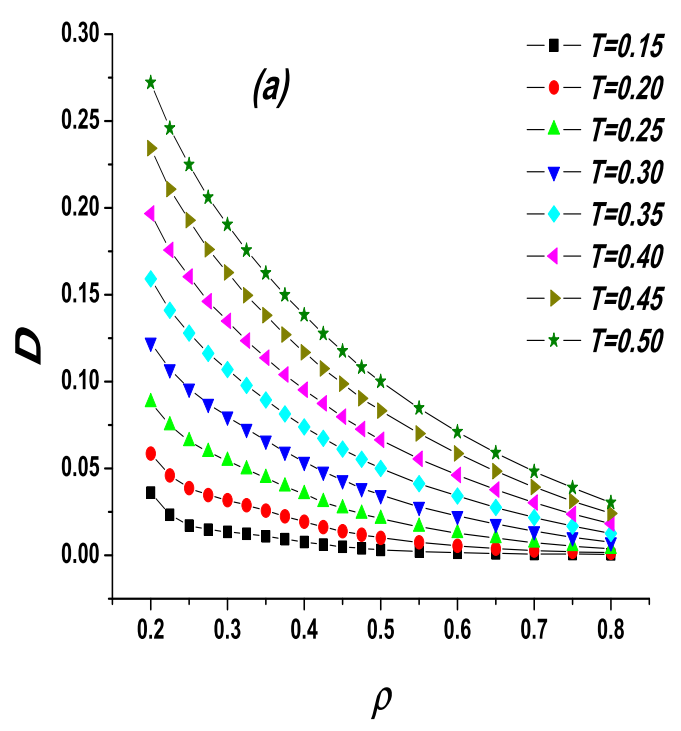

(b)
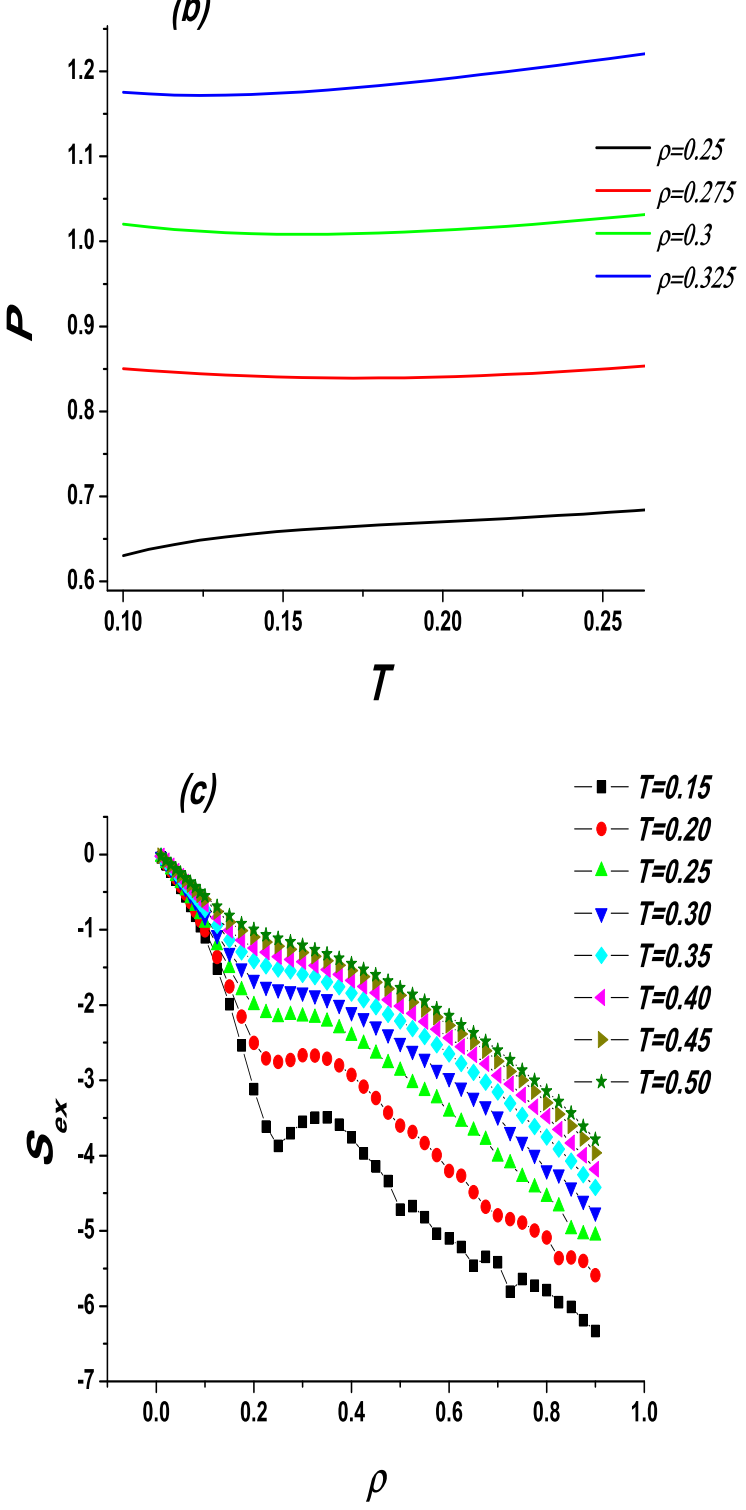

FIG. 5: (Color online). (a) Diffusion coefficient along a set of isotherms; (b) pressure along a set of isochors; (c) excess entropy along a set of isotherms for the system with $\sigma_{1}=1.55$. 


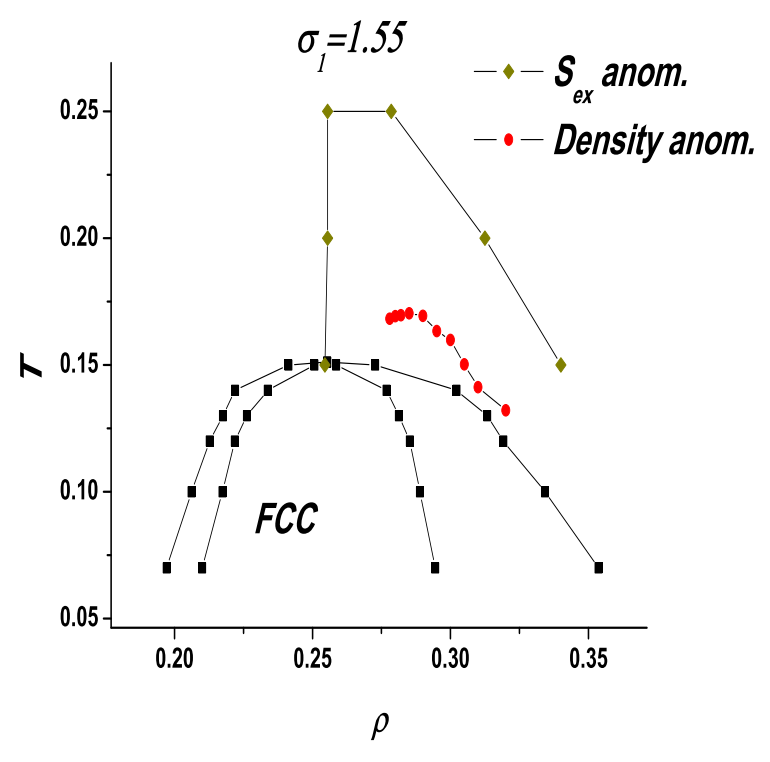

FIG. 6: (Color online). Location of anomalous regions at the phase diagram of the system with $\sigma_{1}=1.55$.

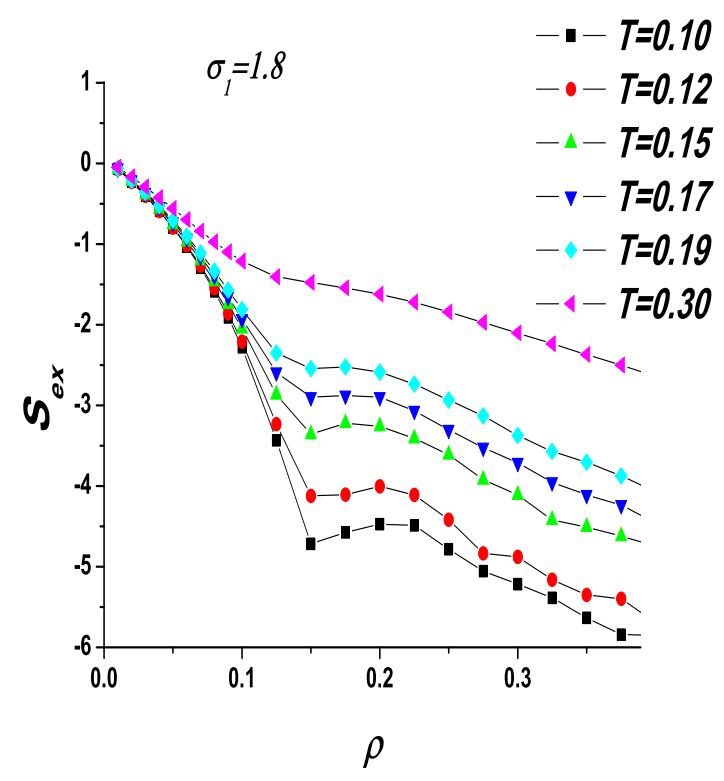

FIG. 7: (Color online). Excess entropy along a set of isotherms for the system with $\sigma_{1}=1.8$.

anomalous increase along low-temperature isotherms (Fig. 7). The situation with density anomaly looks exactly the same as with the diffusion one: the anomalous region shrinks with increasing the step and finally hides under the melting line. At the same time structural anomaly looks very stable with respect to the melting line: it is still located at the temperatures almost twice higher then the melting line maximum (Fig. 8).

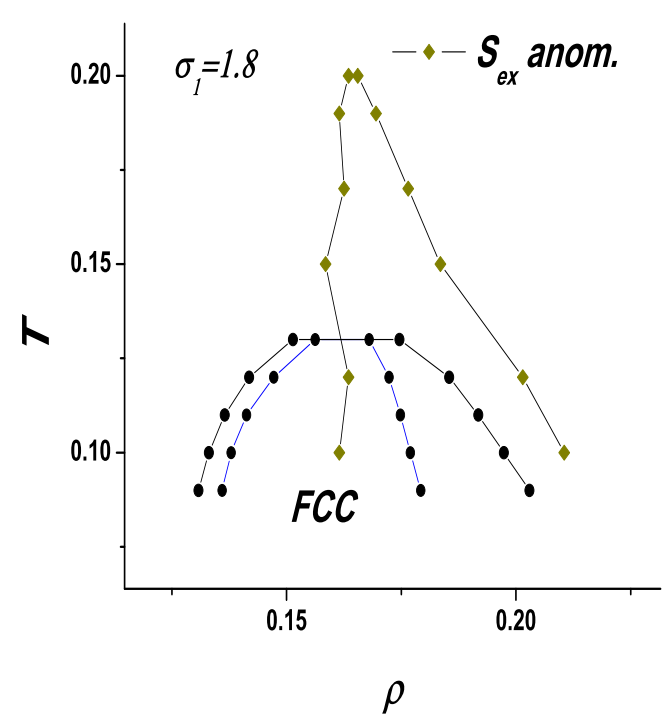

FIG. 8: (Color online). Location of structural anomaly region at the phase diagram of the system with $\sigma_{1}=1.8$. Note that the diffusion and density anomalies are absent for this case.

\section{DISCUSSION}

Figs. 9 (a) and (b) summarize the results for all four systems. One can see that with increasing the step size $\sigma_{1}$ the low density FCC phase and all anomalous regions move to lower densities and lower temperatures. At the same time the maximum temperature of both FCC phase and anomalous regions dramatically drops from $\sigma_{1}=1.35$ to $\sigma_{1}=1.45$ and decreases a bit from 1.45 to 1.55 . However, the rate of decreasing of different curves is different. The diffusion anomaly demonstrates the fastest decay with increasing the repulsive step size. As a result it quickly disappears under the melting line. The density anomaly is the second fastest to decay. As result it disappears somewhere between $\sigma_{1}=1.55$ and $\sigma_{1}=1.8$. The structural anomaly is much more stable than the diffusion and density ones and it does not demonstrate any precursors of disappearance at the moment. It is interesting to note, that similar "resisting" behavior of the structural anomaly was found in Ref. [23], where it was shown that making the soft-core of the potential from Refs. 25, 26] steeper and more penetrable, the regions of density and diffusion anomalies contract in the $T-\rho$ plane, while the region of structural anomaly is weakly affected. The authors of Ref. [23] concluded that a liquid can have anomalous structural behavior without having density or diffusion anomalies. However, in 23] it was not found inversion of the order of anomalies with changing the parameters of the potential. It should be also noted that these results are consistent with results of Ref. 67] where it was proposed that for some of the 

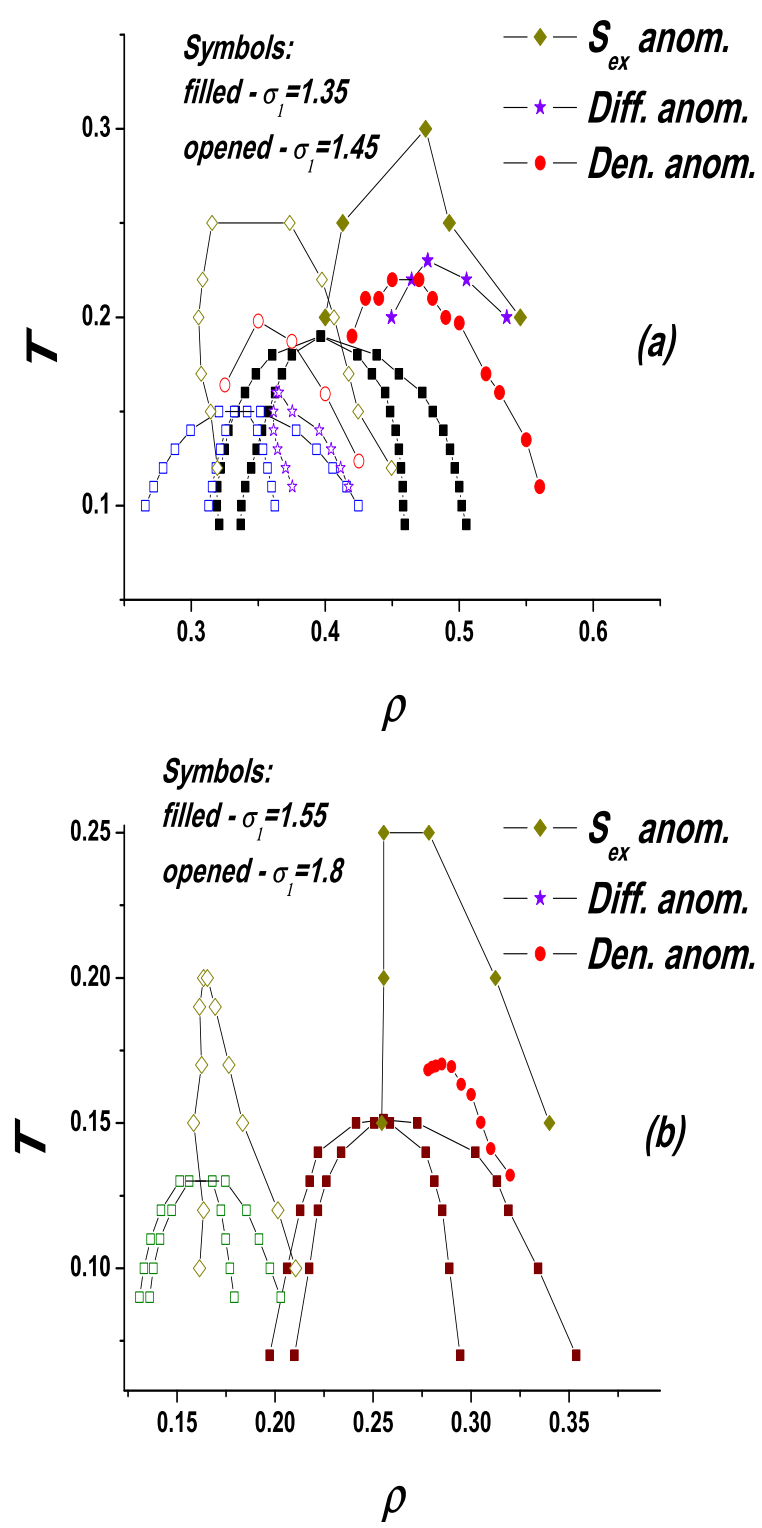

FIG. 9: (Color online). Location of anomalous regions at the phase diagram for (a) systems with $\sigma_{1}=1.35$ and $\sigma_{1}=1.45$; (b) $\sigma_{1}=1.55$ and $\sigma_{1}=1.8$.

core-softened systems anomalies go far under the melting line and become unobservable.

In our previous work we proposed to consider the anomalous regions in scaled coordinates $-\rho \cdot \sigma_{1}^{3}$ and $T / T_{\max }$, where $T_{\max }$ is the temperature of maximum of the melting line of the low density FCC phase. This representation is very useful since it allows to see the relative location of different regions in the phase diagram. Figs. 10 (a) and (b) give such representation for the systems considered in the present paper. This diagram confirms the conclusions of the previous paragraph. Interestingly, the relative height of the structural anomaly even increased from $\sigma_{1}=1.35$ till 1.55. However, further increase to $\sigma_{1}=1.8$ pushes the structural anomaly down to the melting line. One can relate this qualitative change to the fact that the largest step involves in the interaction the second nearest neighbors which alters the system properties. Basing on this change we can not generalize the results to the step of arbitrary width: further increase of the step width will involve more and more neighbors into interaction which can induce more complex effects. In our previous publication we investigated liquid-liquid transition in core-softened systems in frames of perturbation theory [66]. It was shown that the behavior of the system is "periodic" with increasing the step size: liquidliquid transition takes place at some step size, then disappears and on further increase appears again. We can expect that the diffusion and density anomalies will occur again at higher steps, however, this question requires further investigation.

\section{CONCLUSIONS}

In conclusion, this publication represents a detailed study of phase diagrams and anomalous behavior of Smooth Repulsive Shoulder System (SRSS) which belongs to the class of core-softened systems. We investigate the relation of the anomalous behavior with the phase diagram and the interaction potential parameters of the system. We show that both low density crystal phase and all anomalous regions shrink with increasing the step size. However, the rate of the decrease is different. It is also shown that the order of the region of anomalous diffusion and the region of density anomaly is inverted with increasing the width of the repulsive shoulder.

We thank V. V. Brazhkin for stimulating discussions. Y.F. and E.T. also thanks Russian Scientific Center Kurchatov Institute and Joint Supercomputing Center of Russian Academy of Science for computational facilities. The work was supported in part by the Russian Foundation for Basic Research (Grants No 10-02-00694a, 10-0200700 and 11-02-00-341a) and Russian Federal Program 02.740.11.5160.

[1] C.A. Angell, E.D. Finch, and P. Bach, J. Chem. Phys. 65, 3063 (1976).

[2] O. Mishima and H. E. Stanley, Nature 396, 329 (1998).

[3] J. R. Errington and P. G. Debenedetti, Nature (London) 409, 318 (2001).

[4] P.A. Netz, F.V. Starr, H.E. Stanley, and M.C. Barbosa, J. Chem. Phys. 115, 318 (2001).

[5] P. G. Debenedetti, Metastable Liquids: Concepts and Principles (Princeton University Press, Princeton, 1998). 

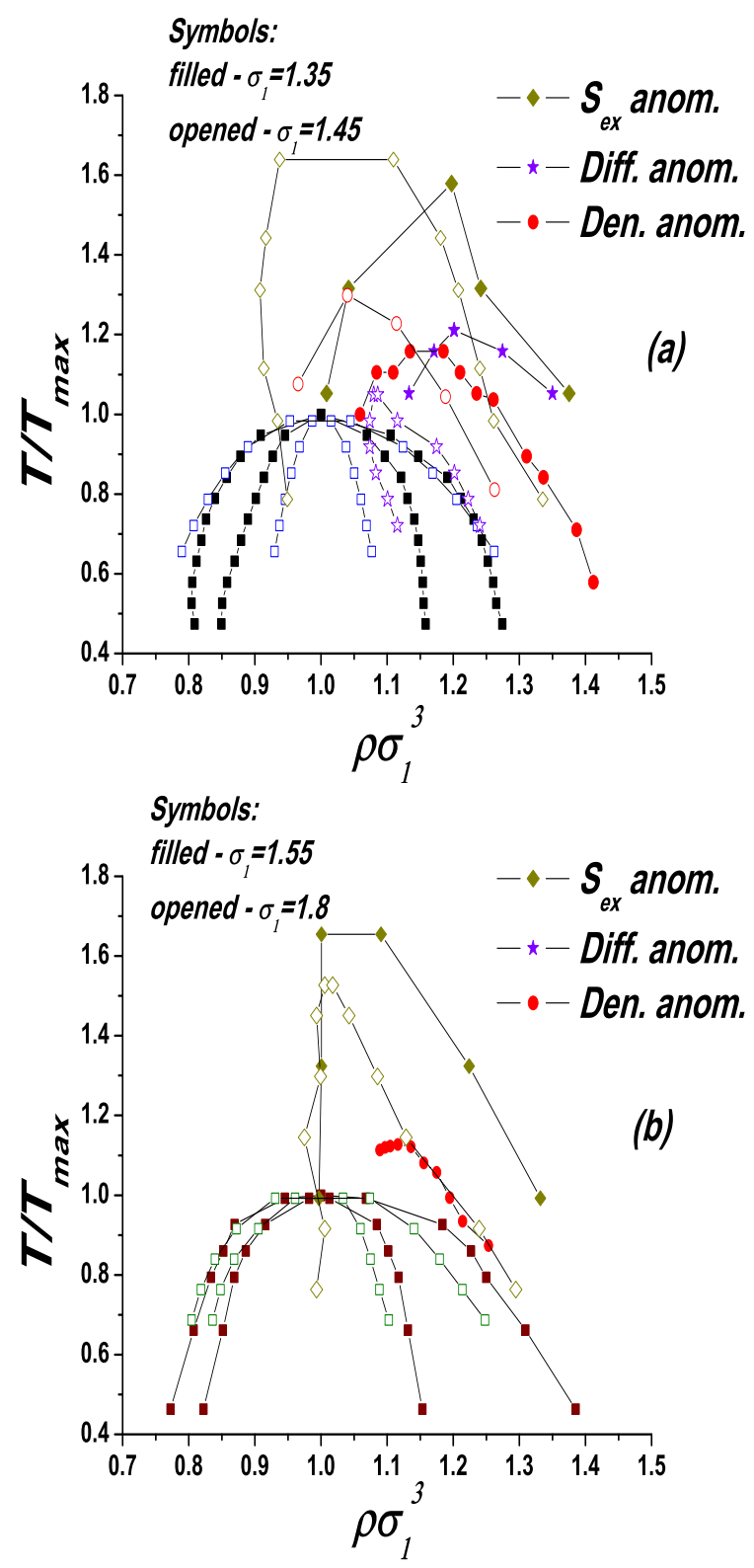

FIG. 10: (Color online). Location of anomalous regions at the phase diagram in scaled coordinates (see the text). (a) $\sigma_{1}=1.35$ and $\sigma_{1}=1.45$; (b) $\sigma_{1}=1.55$ and $\sigma_{1}=1.8$.

[6] V. V. Brazhkin. S. V. Buldyrev, V. N. Ryzhov, and H. E. Stanley [eds], New Kinds of Phase Transitions: Transformations in Disordered Substances [Proc. NATO Advanced Research Workshop, Volga River] (Kluwer, Dordrecht, 2002).

[7] K.K. Mon, N.W. Ashcroft, G.V. Chester, Phys. Rev. B 19, 5103 (1979).

[8] P. Lamparter, S. Stieb, W. Knoll, Z. Naturforsch. A 31, 90 (1976).

[9] H. Thurn, J. Ruska, J. Non-Cryst. Solids 22, 331 (1976).

[10] G.E. Sauer, L.B. Borst, Science 158, 1567 (1967).

[11] S.J. Kennedy, J.C. Wheeler, J. Chem. Phys. 78, 1523 (1983)

[12] J.F. Wax, R. Albaki, J.L. Bretonnet, Phys. Rev. B 62,
14818 (2000)

[13] T. Tsuchiya, J. Phys. Soc. Jpn 60, 227 (1991).

[14] C.A. Angell, R.D. Bressel, M. Hemmatti, E.J. Sare, J.C. Tucker, Phys. Chem. Chem. Phys. 2, 1559 (2000).

[15] M.S. Shell, P.G. Debenedetti, A.Z. Panagiotopoulos, Phys. Rev. E 66, 011202 (2002).

[16] P.H. Poole, M. Hemmati, C.A. Angell, Phys. Rev. Lett. 79, 2281 (1997).

[17] S. Sastry, C.A. Angell, Nature Mater. 2, 739 (2003).

[18] V.V. Brazhkin, E.L. Gromnistkaya, O.V. Stalgorova, A.G. Lyapin, Rev. High Pressure Sci. Technol. 7, 1129 (1998).

[19] Y. Katayama, T. Mizutani, W. Utsumi, O. Shimomura, M. Yamakata, K. Funakoshi, Nature (London) 403, 170 (2000).

[20] Y. Katayama, Y. Inamura, T. Mizutani, W. Yamakata, M. Utsumi, S.O., Science 306, 848 (2004).

[21] Pol Vilaseca and Giancarlo Franzese, Journal of NonCrystalline Solids 357, 419 (2011).

[22] S. V. Buldyrev, G. Malescio, C. A. Angell, N. Giovambattista, S. Prestipino, F. Saija, H. E. Stanley and L. Xu, J. Phys.: Condens. Matter 21, 504106 (2009).

[23] Pol Vilaseca and Giancarlo Franzese, J. Chem. Phys., 133, 084507 (2010).

[24] G. Franzese, G. Malescio, A. Skibinsky, S.V. Buldyrev, and H. E. Stanley, Nature 409, 692 (2001).

[25] G. Franzese, J. Mol. Liq. 136, 267 (2007).

[26] A. B. de Oliveira, G. Franzese, P. A. Netz, and M. C. Barbosa, J. Chem. Phys. 128, 064901 (2008).

[27] J. R. Errington, Th. M. Truskett, J. Mittal, J. Chem. Phys. 125, 244502 (2006).

[28] J. Mittal, J. R. Errington, Th. M. Truskett J. Chem. Phys. 125, 076102 (2006).

[29] P. Kumar, L. Xu, Z. Yan, M. G. Mazza, S. V. Buldyrev, S. H. Chen, S. Sastry, and H. E. Stanley, Phys. Rev. Lett., 97, 177802 (2006).

[30] P. C. Hemmer and G. Stell, Phys. Rev. Lett. 24, 1284(1970).

[31] G. Stell and P. C. Hemmer, J. Chem. Phys. 56, 4274 (1972).

[32] G. Malescio, J. Phys.: Condens. Matter 19, 07310 (2007).

[33] E.Velasco, L. Mederos, G. Navascues, P. C. Hemmer, and G. Stell, Phys. Rev. Lett. 85, 122 (2000).

[34] P. C. Hemmer, E.Velasko, L. Mederos, G. Navascues, and G. Stell, J. Chem. Phys. 114, 2268 (2001).

[35] M. R. Sadr-Lahijany, A. Scala, S. V. Buldyrev and H. E. Stanley, Phys. Rev. Lett. 81, 4895 (1998).

[36] M. R. Sadr-Lahijany, A. Scala, S. V. Buldyrev and H. E. Stanley, Phys. Rev. E 60, 6714 (1999).

[37] P. Kumar, S. V. Buldyrev, F. Sciortino, E. Zaccarelli, and H. E. Stanley, Phys. Rev. E 72, 021501 (2005).

[38] L. Xu, S. V. Buldyrev, C. A. Angell, and H. E. Stanley, Phys. Rev. E 74, 031108 (2006).

[39] E. A. Jagla, J. Chem. Phys. 111, 8980 (1999); E. A. Jagla, Phys. Rev. E 63, 061501 (2001).

[40] F. H. Stillinger and D. K. Stillinger, Physica (Amsterdam) 244A, 358 (1997).

[41] A. B. de Oliveira, P. A. Netz, T. Colla, and M. C. Barbosa, J. Chem. Phys. 124, 084505 (2006).

[42] A. B. de Oliveira, P. A. Netz, T. Colla, and M. C. Barbosa, J. Chem. Phys. 125, 124503 (2006).

[43] P. A. Netz, S. Buldyrev, M. C. Barbosa and H. E. Stanley, Phys. Rev. E 73, 061504 (2006).

[44] A. B. de Oliveira, M. C. Barbosa, and P. A. Netz, Physica 
A 386, 744 (2007).

[45] J. Mittal, J. R. Errington, and T. M. Truskett, J. Chem. Phys. 125, 076102 (2006).

[46] H. M. Gibson and N. B. Wilding, Phys. Rev. E 73, 061507 (2006).

[47] P. Camp, Phys. Rev. E 71, 031507 (2005).

[48] A. B. de Oliveira, G. Franzese, P. A. Netz, and M. C. Barbosa, J. Chem. Phys. 128, 064901 (2008).

[49] L. Xu, S. Buldyrev, C. A. Angell, and H. E. Stanley, Phys. Rev. E 74, 031108 (2006).

[50] A. B. de Oliveira, P. A. Netz, and M. C. Barbosa, Euro. Phys. J. B 64, 481 (2008).

[51] Z. Yan, S. V. Buldyrev, N. Giovambattista, and H. E. Stanley, Phys. Rev. Lett. 95, 130604 (2005).

[52] P.H. Poole, F. Sciortino, U. Essmann, and H.E. Stanley, Nature 360, 324 (1992).

[53] L. Xu, P. Kumar, S.V. Buldyrev, S.-H. Chen, P.H. Poole, F. Sciortino, and H.E. Stanley, PNAS 102, 16558 (2005).

[54] P. Kumar, S.V. Buldyrev, S.R. Becker, P.H. Poole, F.W. Starr, and H.E. Stanley, PNAS 104, 9575 (2007).

[55] G. Franzese and H. E. Stanley, J. Phys.: Condens. Matter 19, 205126 (2007).

[56] A. B. de Oliveira, E. B. Neves, C. Gavazzoni, J. Z. Paukowski, P. A. Netz, and Marcia C. Barbosa, J. Chem. Phys. 132, 164505 (2010).
[57] Yu. D. Fomin, N. V. Gribova, V. N. Ryzhov, S. M. Stishov and Daan Frenkel, J. Chem. Phys., 129, 064512 (2008).

[58] Yu. D. Fomin, E.N. Tsiok and V.N. Ryzhov, J. Chem. Phys. 134, 044523 (2011).

[59] N. V. Gribova, Yu. D. Fomin, Daan Frenkel, V. N. Ryzhov, Phys. Rev. E 79, 051202 (2009).

[60] Yu. D. Fomin, V. N. Ryzhov, N. V. Gribova, Phys. Rev. E 81, 061201 (2010).

[61] Yu. D. Fomin and V. N. Ryzhov, Phys. Lett. A 375, 2181 (2011).

[62] Yu. D. Fomin, E.N. Tsiok and V.N. Ryzhov, arXiv:1107.2799 (2011).

[63] M. S. Shell, P. G. Debenedetti, and A. Z. Panagiotopoulos, Phys. Rev. E 66, 011202 (2002).

[64] Daan Frenkel and Berend Smit, Understanding molecular simulation (From Algorithms to Applications), 2nd Edition (Academic Press), 2002.

[65] E. A. Koopman and C.P. Lowe, J. Chem. Phys. 124, 204103 (2006)

[66] Yu.D. Fomin, V.N. Ryzhov, E.E. Tareeva, Phys. Rev. E 74, 041201 (2006).

[67] A.B. de Oliveira, P.A. Netz and M.C. Barbosa, Europhys. Lett. 85, 36001 (2009). 